\title{
Refractive and diffractive properties of planar micro-optical elements
}

\author{
M. Rossi, R. E. Kunz, and H. P. Herzig
}

\begin{abstract}
The refractive and the diffractive properties of planar micro-optical elements are investigated. The transition between purely refractive and purely diffractive planar microlenses is numerically simulated for the example of differently designed phase-matched Fresnel elements. Results obtained from numerical simulations and experiments show that the refractive and diffractive types exhibit a distinctly different behavior in the presence of small fabrication errors or wavelength deviations. Based on these results, design rules for various applications, including low- and high-numerical-aperture lenses and hybrid refractive-diffractive elements, are derived. For a high-numerical-aperture $(f / \#=1.0)$ lens the experimental characterization of the irradiance distribution in the image space is presented and shown to agree well with theoretical predictions.
\end{abstract}

\section{Introduction}

Classical lenses and mirrors consisting of macroscopic surface-relief structures are designed by use of the laws of geometrical optics, which treat light propagation by the refraction and the reflection of geometrical rays at optical interfaces. The eikonal equation as the basis of geometrical optics can be derived from Maxwell's equations in the limit where the wavelength tends to zero. ${ }^{1}$ Therefore no wavelengthdependent properties, apart from those that are due to material dispersion are observed. However, owing to the large structure size, these elements are often not appropriate for the integration in optical and optoelectronical microsystems. One possibility for miniaturization is the classical Fresnel lens, obtained by a simple dissection of the geometrically calculated profile. Although the eikonal equation yields a locally correct phase distribution, the laws of geometrical optics do not consider the interference effects of the waves emanating from the different segments. Owing to the resulting incoherent superposition of different waves, the resolution of a classical Fresnel lens is limited by the size of individual segments.

\footnotetext{
M. Rossi and R. E Kunz are with the Paul Scherrer Institute, Badenerstrasse 569, Zurich CH-8048, Switzerland. H. P. Herzig is with the Institute of Microtechnology, University of Neuchâtel, Neuchâtel, Switzerland.

Received 3 January 1995.

0003-6935/95/265996-12\$06.00/0.

(C) 1995 Optical Society of America.
}

In contrast, holographic optical elements and diffractive optical elements (DOE's) do consider these effects. In general, DOE's are designed based on the wave nature of light, expressed by the laws of wave optics. An example of such an element is the Fresnel zone plate. The diffraction-limited focusing function of these binary phase or amplitude gratings can be explained only by the coherent superposition of different waves. Because diffraction effects are strongly dependent on the wavelength of light, the imaging properties of DOE's typically suffer from severe chromatic aberrations. To obtain a high efficiency in one or several specific grating diffraction orders, DOE's require a precisely controlled phase profile.

Therefore the optical properties of planar microoptical elements consisting of grating structures combined with continuous surface-relief profiles between the grating lines are of great interest. Depending on the sizes of the surface-relief features, the laws of geometrical optics can also be adequate for the description of their behavior. Our purpose is to show under which conditions the effects of refraction and diffraction are dominant. We especially concentrate on the consequences of typical fabrication inaccuracies and wavelength deviations and give some fabricationrelated design rules.

The analysis is carried out on phase-matched Fresnel elements ${ }^{2}$ (PMFE's) because they offer a generalized approach for the design and the fabrication of planar micro-optical elements. In particular they offer degrees of freedom for choosing the weights of the refractive and the diffractive contributions to the 
overall optical properties. In Section 2 we outline the concept of the PMFE design, which is based on both geometrical and wave optics. The mathematical models for the numerical simulation of the refractive and the diffractive properties of PMFE's are introduced in Section 3. In Section 4 the optical behavior of differently designed planar micro-optical elements in the presence of fabrication errors and illumination-wavelength deviations are treated for examples of lenses with numerical apertures NA = $0.05(f / \#=10)$ and NA $=0.45(f / \#=1.0)$. In order to demonstrate the feasibility of the PMFE design and fabrication, we present an experimental spot characterization on an $\mathrm{NA}=0.45$ lens. As a special combination of refraction and diffraction, the limits of operation for a hybrid achromat are derived.

\section{Phase-Matched Fresnel-Element Concept}

For the combination of refractive and diffractive properties a general approach for the design is chosen. The following discussions concentrate on imaging elements; however, the PMFE concept is not limited to this class of elements. We start with the statement that a PMFE is equivalent to a segmented microrelief structure. ${ }^{2}$

Each segment acts as a transmissive or a reflective facet; thus an incoming primary wave $u_{0}$ is split into secondary wavelets $u_{j}$. In a first approximation we do not consider the calculation of the exact mathematical form of the secondary wavelets $u_{j}$, which would require rigorous diffraction theory. The surfacerelief profile of each segment is designed to perform the desired optical function in the sense of geometrical optics. An equivalent formulation of this condition is that the optical path length $W_{j}($ Ref. 3 ) between the object and the image points for any ray $r_{j}$ that crosses the segment $\sigma_{j}$ is constant (cf. Fig. 1). For the calculation of the optical path length $W_{j}$ the dispersive refractive indices $n_{0}, n^{\prime}$, and $n$ in the object space, the image space, and the PMFE layer, respectively, have to be taken into account. In this paper, $n_{0}$ and $n^{\prime}$ are set to the constant value of 1 . The size and the relative arrangement of the individual segments are determined by additional design requirements and constraints that originate from fabrication techniques.

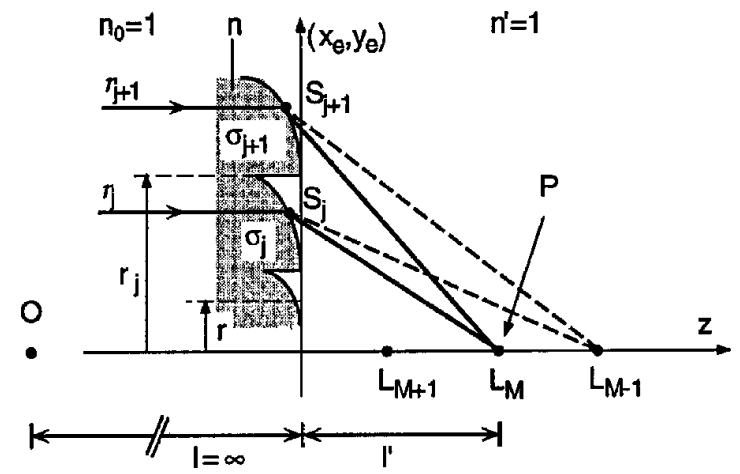

Fig. 1. Definitions of PMFE segments and focal points of different orders.
For the calculation of the segment boundaries the wave nature of light is taken into account. In the sense of a modified Huygens-Fresnel principle, which gave rise to the use of Fresnel in the term phasematched Fresnel elements, the amplitude of the optical field in the far field is given by the superposition of all secondary wavelets $u_{j}$ (Ref. 2 ). In order to take advantage of interference effects (be it constructive or destructive) of these wavelets, we establish a controlled phase relation between the wavelets emanating from all segments, using phase-sensitive raytracing algorithms ${ }^{3}$ with the concept of geometrical wave fronts. As an example, we consider the imaging of a point source $\mathrm{O}$ into an image point $\mathrm{P}$, where the object distance $l$ is taken to be infinite, as shown in Fig. 1. This is achieved by choice of the segment boundaries in a manner that the optical phase difference $\Delta W_{j}$ in $L^{\prime}=P$ of two rays $r_{j}$ and $r_{j+1}$, crossing neighboring segments $\sigma_{j}$ and $\sigma_{j+1}$, is an integer multiple $M_{j}$ of $2 \pi$ (cf. Fig. 1). The equivalent expression for the optical path length is

$$
\Delta W_{j}=W\left(\overline{\mathrm{OS}_{j+1} \mathrm{P}}\right)-W\left(\overline{\mathrm{OS}_{j} \mathrm{P}}\right)=M_{j} \lambda,
$$

where $\lambda$ is the wavelength in air. The fact that the integer $M_{j}$, called the phase-matching number, may be locally different from one boundary to another is an important degree of freedom for the design of these elements.

Because the approach presented in the previous paragraph is a rather general one, the width $w_{j}$ and the depth $h_{j}$ of the segments and the exact form and position of their boundaries are not uniquely determined. Specific design strategies are characterized by the alignment of the segments and the local values of the phase-matching number $M_{j}$. The choice of the alignment of the segments, e.g., top or bottom aligned (cf. Fig. 2), is influenced by the fabrication technology and becomes relevant in the high-NA regions of planar lenses. The value of $M_{j}$ determines the width and the depth of the segments. One possible strategy is to choose $M_{j}$ so that the segment width $w_{j}$ remains above a minimal value $w_{\min }$ for a given fabrication technology. ${ }^{4}$ This special case corresponds to the approach used by Futhey ${ }^{5}$ in the design of superzone diffractive lenses and by Marron et al. ${ }^{6}$ for their higher-order kinoforms. Besides the aspect of segment width and depth, the refractive and the diffractive properties are a function of the number of illuminated segments. With the variation of $M_{j}$ the element can behave in the two extreme cases as a purely diffractive $\left(M_{j}=M=1\right.$ with the number of illuminated segments $Q \gg 1$ ) or refractive (with $M$ large enough to obtain only one segment, i.e., $Q=1$ ) element.

The design procedure presented here, which locally optimizes the surface relief, does not rely on any approximations other than the validity of the scalar theory. Particularly, good results can be obtained for lenses with a high $\mathrm{NA}^{4}$ because the calculations are not restricted to the paraxial domain. ${ }^{7}$ Because of the direct calculation of a locally optimized surface 


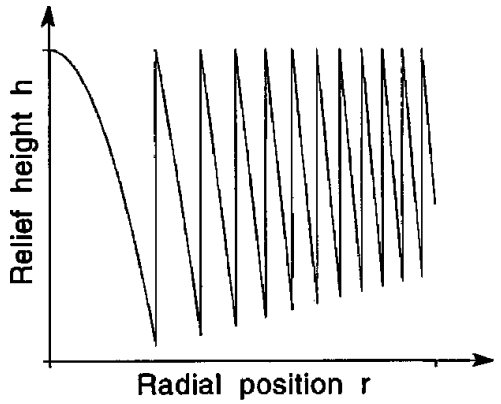

(a)

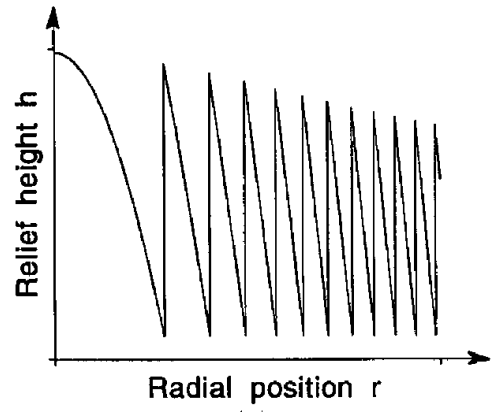

(b)

Fig. 2. Schematic design examples for (a) top-aligned and (b) bottom-aligned PMFE microstructures.

relief instead of a phase function in a plane, no complex corrections for the profile are required as, e.g., in Ref. 8.

The approach of using a segmented surface-relief structure for interfering and phase matching the secondary wavelets in the image space can also be interpreted as representing a miniature implementation of an optical phased array. ${ }^{9}$

The PMFE microstructures can be fabricated by any technology that enables one to produce the segments with a sufficient accuracy. The segments can be formed as continuous surface reliefs by means of direct laser ${ }^{10}$ or electron-beam writing, ${ }^{11}$ or as binary optical $^{12}$ structures. In this paper we concentrate on elements fabricated by direct laser writing in photoresist.

\section{Theoretical Description}

For the theoretical description of the PMFE behavior two different models are used. In a first approach the diffraction patterns are calculated in the Fresnel diffraction regime. These calculations give reliable results for lenses with NA $\leq 0.05(f / \# \geq 10)$ (Ref. 7) and give a good intuition for the basic processes. More precise results can be obtained from the subsequent calculations in the Rayleigh-Sommerfeld approximation. These numerical simulations take also into account the finite thickness of the PMFE surface relief and determine the diffraction pattern of the structures designed by use of the algorithms in Section 2.

The PMFE concept combines geometrical and wave optics in a way that the segment boundary pattern acts locally as a diffraction grating having the $M_{j}$ th- order focus at the position $l^{\prime}=L_{M}$ and that the segment relief shape is optimized for the geometrical optical refraction of light rays into the point $L^{\prime}$. Besides this main focal point $L^{\prime}$ of the diffraction grating, there exist additional positions $L_{N}$ on the $z$ axis where the optical-path difference $\Delta W_{j}$ is an integer multiple $N$ of $\lambda$ with $N>M_{j}$ or $N<M_{j}$ (cf. Fig. 1). We can obtain the values $L_{N}$ for these higher and lower diffraction orders by solving Eq. (1) with $M_{j}$ replaced by $N$. A plot of these positions $L_{N}$ for lenses with NA $=0.05$ and different values of $M_{j}$ is given in Fig. 3. The density of higher and lower diffraction orders on the optical axis apparently rises with increasing values of $M_{j}$ and is an important factor for the behavior of planar micro-optical elements in the presence of surface distortions and wavelength deviations.

Whereas the above calculations determine the positions where constructive interference of the different secondary wavelets $u_{j}$ occurs, the surface-relief profile of the segments determines the amount of light at these positions. A first estimation of the intensity distribution among these various diffraction orders can be obtained if we assume that a paraxial description $^{7,13}$ is valid. In this case, Eq. (1) can be transformed to

$$
r_{j}^{2}=2 j M_{j} \lambda_{0} l^{\prime},
$$

where $r_{j}$ is the distance of the segment boundary from the origin and $\lambda_{0}$ is the design wavelength in air. The phase shift introduced by the continuous surfacerelief segments at an arbitrary position $r$ is approximated by a quadratic function.

$$
\Phi_{j}(r)=\alpha M_{j} 2 \pi\left(j-\frac{r^{2}}{2 M_{j} \lambda_{0} l^{\prime}}\right), \quad r_{j} \leq r \leq r_{j+1},
$$

where the parameter

$$
\alpha=\frac{\lambda_{0}\left[n\left(\lambda_{1}\right)-1\right]}{\lambda_{1}\left[n\left(\lambda_{0}\right)-1\right]}
$$

quantifies the phase delay for an illumination wavelength $\lambda_{1} \neq \lambda_{0}$ (cf. Ref. 7). In addition to chromatic

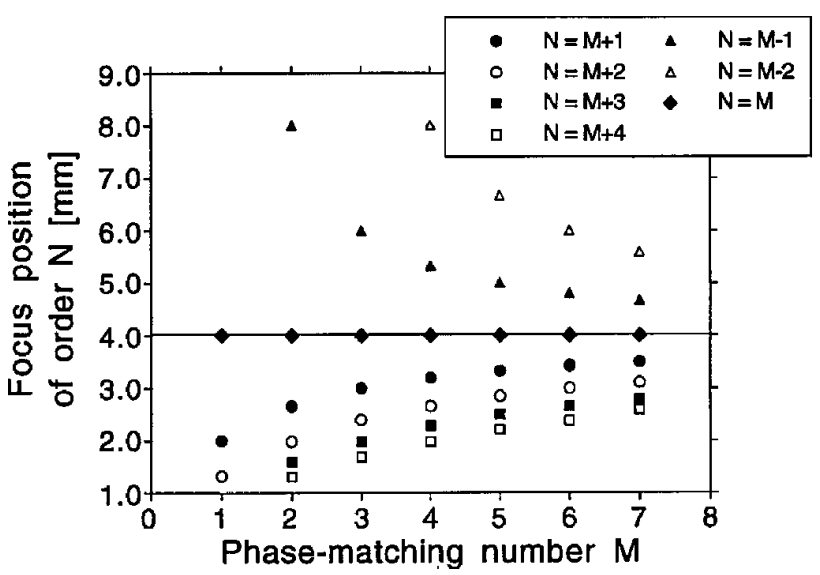

Fig. 3. Axial position of higher- and lower-order foci for PMFE's with different phase-matching numbers. 
effects we also treat the influence of fabrication inaccuracies in the form of relief-profile scaling errors. A typical distortion of an original surface-relief profile $h(r)$ is expressed by a term $\mu$ as

$$
h^{\prime}(r)=\mu h(r) .
$$

Because the required phase shift is realized by a surface-relief profile with the relation

$$
h(r)=\frac{\lambda_{0}}{2 \pi\left[n\left(\lambda_{0}\right)-1\right]} \Phi(r),
$$

we also introduce the multiplicative factor $\mu$ in Eq. (3) for modeling relief-height errors.

We now introduce a new variable $\xi=r^{2} / 2 \lambda_{0} l^{\prime}$. The segment transmission function $t(\xi)=\exp [i \Phi(\xi)]$ is then periodically extended and describes a Fresnel lens with an infinite number of segments and a constant phase-matching number $M_{j}=M . \quad t(\xi)$ is a periodic function with a period $T=1$ and can be approximated by a Fourier series

$$
\begin{aligned}
t(\xi)= & \sum_{N=-\infty}^{\infty} \exp [-i \pi(\alpha \mu M-N)] \operatorname{sinc}(\alpha \mu M-N) \\
& \times \exp (-i 2 \pi N \xi)
\end{aligned}
$$

or

$$
\begin{aligned}
t(r)= & \sum_{N=-\infty}^{\infty} \exp [-i \pi(\alpha \mu M-N)] \operatorname{sinc}(\alpha \mu M-N) \\
& \times \exp \left[-\frac{i \pi N r^{2}}{M \lambda_{0} l^{\prime}}\right]
\end{aligned}
$$

where the sinc function is defined as

$$
\operatorname{sinc}(x)=\frac{\sin (\pi x)}{\pi x} .
$$

Expansion (8) requires a large number of segments $j$ for being valid. The following numerical simulations in Subsections 4.B. and 4.C. indicate that, already for four illuminated segments, the optical behavior of a microlens can be described by Eq. (8) with a good accuracy. As a comparison, the transmission function for a lens with only one segment, designed for a focal length $f$ and a wavelength $\lambda_{1}$, is given by ${ }^{14}$

$$
t(r)=\exp \left(-\frac{i \pi r^{2}}{\lambda_{1} f}\right)
$$

This equation describes a purely refractive lens with one single focal point and a wavelength dependence given by the material dispersion $n(\lambda)$ [cf. Eq. (6)]; we have set $n_{0} \equiv 1$ and $n^{\prime} \equiv 1$. If we compare Eqs. (8) and (10), the transmission function of a paraxial Fresnel lens can then be interpreted as a summation over the transmission functions of a series of lenses with focal lengths

$$
l_{N}=\frac{M l^{\prime}}{N} \frac{\lambda_{0}}{\lambda_{1}}
$$

The normalized energy in these focal points is proportional to the squared modulus of the Fourier coefficient:

$$
\eta_{N}=\operatorname{sinc}^{2}(\alpha \mu M-N) .
$$

If the illumination wavelength $\lambda_{1}$ is not equal to the design wavelength $\lambda_{0}$, the position of the focal points $l_{N}$ and their efficiency $\eta_{N}$ change according to Eqs. (11) and (12) A profile-height error $(\mu-1) \neq 0$ does not affect the position of the foci $l_{N}$ but alters the energy distribution among them. From the sinc function in Eq. (12) follows that only two diffraction orders $N$ simultaneously can have an efficiency of more than $5 \%$, independent of the values of $\alpha, \mu$, and $M$. This fact that only two values of $\eta_{N}$ are in the central sinc lobe is similar to the behavior of blazed gratings working in higher diffraction orders, ${ }^{15}$ which can be interpreted as special, nonfocusing cases of PMFE's.

As a further consequence of Eq. (12), the probability that an order other than the designed diffraction order $M$ is the most efficient one, i.e., $\eta_{N}>\eta_{M}$ for $N \neq$ $M$, increases for higher values of $M$. This can also be explained with refraction-based considerations. Because the higher and lower diffraction orders $N$ move nearer to the nominal focal point $L^{\prime}$ for higher values of $M$ (cf. Fig. 3), it takes smaller profile deviations to refract the light at these positions $L_{N}$ of constructive interference and therefore to increase their efficiency $\eta_{N}$.

The existence of additional focal points and their behavior under wavelength or fabrication inaccuracies are the most important differences in the properties of purely refractive and diffractive lenses. The following investigations concentrate on these effects in the transition region between the two extremes. As a consequence, we encounter cases in which the assumption of a high number of segments $j$ in Eq. (8) is no longer fulfilled. In addition, these paraxial equations are not suited for the numerical simulation of high-NA lenses. Therefore the Rayleigh-Sommerfeld diffraction formulas ${ }^{16}$ are used here for the quantitative evaluation of these PMFE diffraction patterns:

$$
\begin{aligned}
u_{2}(x, y)= & \iint_{-\infty}^{\infty} u_{1}\left(x_{e}, y_{e}\right)\left(\frac{z_{12}}{i \lambda r_{12}^{2}}\right) \exp \left(j k r_{12}\right) \mathrm{d} x_{e} \mathrm{~d} y_{e} \\
= & \iint_{-\infty}^{\infty} u_{1}\left(x_{e}, y_{e}\right) \\
& \times \frac{\exp \left\{i k z_{12}\left[1+\left(\frac{x-x_{e}}{z_{12}}\right)+\left(\frac{y-y_{e}}{z_{12}}\right)^{2}\right]^{1 / 2}\right\}}{i \lambda z_{12}\left[1+\left(\frac{x-x_{e}}{z_{12}}\right)+\left(\frac{y-y_{e}}{z_{12}}\right)^{2}\right]} \\
& \times \mathrm{d} x_{e} \mathrm{~d} y_{e} .
\end{aligned}
$$


The variable $u_{1}\left(x_{e}, y_{e}\right)$ is the complex-amplitude distribution in the tangential plane $\left(x_{e}, y_{e}\right)$ to the microoptical surface relief (cf. Fig. 1) and is calculated by our taking into account both the phase shift of the PMFE relief and the refraction of the rays at the curved surface. With this equation the finite depth of the surface-relief profile is correctly taken into account. The complex-amplitude distribution in some plane $(x, y)$, that fulfills the Rayleigh-Sommerfeld conditions $\left(z_{12} \gg \lambda\right)$ is given by $u_{2}(x, y) ; z_{12}$ is the distance of the planes $\left(x_{e}, y_{e}\right)$ and $(x, y)$. Because the radial distribution of the relief data points resulting from the PMFE design is dependent on the local profile structure, we did not make use of fast Fourier algorithms for the evaluation of Eq. (13) and solved it by direct numerical integration.

\section{Experiments and Numerical Simulations on the Refractive-Diffractive Behavior}

\section{A. General Remarks}

In Section 2 it was shown that the phase-matching number $M_{j}$ and the strategy of its variation over the element surface are the most important features of the design procedure for PMFE's. The value $M_{j}\left(x_{e}, y_{e}\right)$ determines essentially the width and the depth of the microlens segments. One important way of using this parameter is the adaptation of the segment size to the limits imposed by the fabrication technology. Moreover, the optical characteristic of microlenses also can depend on the phase-matching number. Especially when small fabrication errors occur or when the micro-optical element is illuminated by a wave with a wavelength $\lambda_{1} \neq \lambda_{0}$, where $\lambda_{0}$ is the design wavelength, the irradiance distribution and the efficiency of the microlenses depends on the number and the size of the illuminated segments. We first show some basic effects concerning the refractive and the diffractive behavior of planar microlenses at representative examples. At the end of this section we give a summary and list some design rules for typical applications.

The following numerical simulations were performed on a series of eight different PMFE's, which focus a collimated $\mathrm{He}-\mathrm{Ne}$ laser beam (wavelength $\lambda_{\mathrm{He}-\mathrm{Ne}}=\lambda_{0}=632.8 \mathrm{~nm}$ ) at a distance $l^{\prime}=4.0 \mathrm{~mm}$. These lenses have a numerical aperture NA $=0.05$ $(f / \#=10)$; their phase-matching numbers $\left(M_{j}=M=\right.$ constant) are varied from $M=1$ to $M=8$. The PMFE with $M=1$ consists of $Q=8$ segments (cf. Fig. 4 ), and because of the $2 \pi$ phase steps, it represents the purely diffractive case. ${ }^{17}$ Owing to the clamped finite aperture, the case of a purely refractive microlens is reached by the element with $M=8$. According to the increasing size of contiguous surface-relief areas and the decreasing number of segment boundaries, the elements with phasematching numbers in the range from $M=2$ to $M=7$ can be described as having both refractive and diffractive attributes. The element with $M=2$ has $Q=4$ complete segments; the one with $M=4$ has $Q=2$ complete segments. The PMFE's with $M=5$ to $M=7$ all include
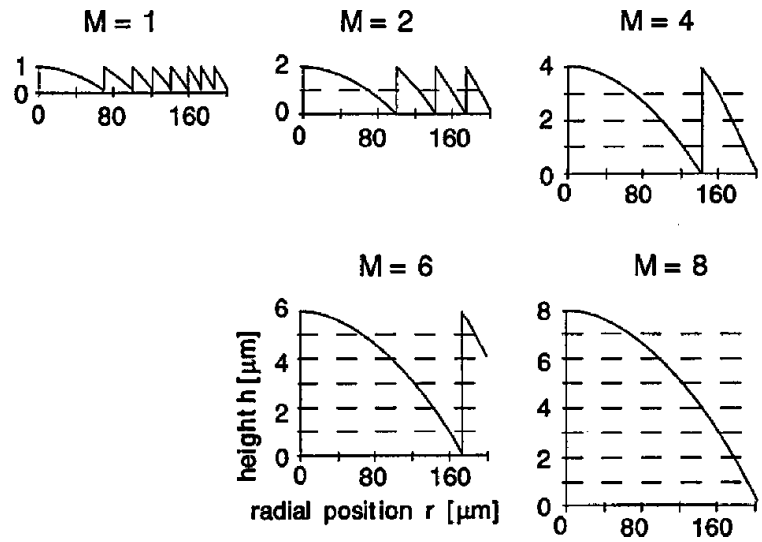

Fig. 4. PMFE surface-relief elements for numerical simulations.

exactly one segment boundary, and the area of the second, outer segment decreases relative to the area of the inner segment. All these PMFE's are compatible with fabrication technologies such as laser-beam writing, ${ }^{10}$ although resist films thicker than $5 \mu \mathrm{m}$ require nonstandard processing.

\section{B. Monochromatic Applications}

Provided that the wavelength $\lambda_{1}$ of the monochromatic illumination wave is equal to the design wavelength $\lambda_{0}$, the microlens performance is determined mainly by the fabrication accuracy. The lateral pattern of the microlenses, i.e., the position of the segment boundaries, can be realized with very small tolerances by use of photolithographic fabrication techniques such as laser or electron-beam writing. Interferometric control of the writing stages and modern mask aligners that are used for binary optics guarantee a very high lateral precision for the segment boundaries. The vertical shape of the surfacerelief profile is more difficult to fabricate with a good reliability. For the fabrication of the elements by direct laser-beam writing, relief-height errors in the range of several percent, owing to irregularities of the photoresist films, the exposure dose, and the wet etching process, are typical. We analyze the consequences of surface-relief errors by considering elements whose original relief depth is multiplied by a constant factor $\mu$.

In order to demonstrate the most important effects, we chose a relatively high surface-relief error of $10 \%$ $(\mu=0.9$. The results obtained with the RayleighSommerfeld integrals are shown in Figs. 5(a)-5(e). For each microlens the irradiance on the optical axis and the energy encircled by a disk with the Airy radius $q_{a}$ of the undistorted lens $\left(q_{a} \approx 1.22 l^{\prime} \lambda_{0} /\right.$ $2 a \approx 7.7 \mu \mathrm{m}$, with lens aperture $2 a=400 \mu \mathrm{m}$ ) are plotted as a function of the distance $z_{12}$ from the micro-optical element on the optical axis. Irradiance and energies of the distorted PMFE's were normalized by the corresponding values obtained for $\mu=1.0$. For this ideal case, all different design variants showed identical diffraction patterns. The bold vertical lines in Figs. 5(a)-5(d) indicate the position and 


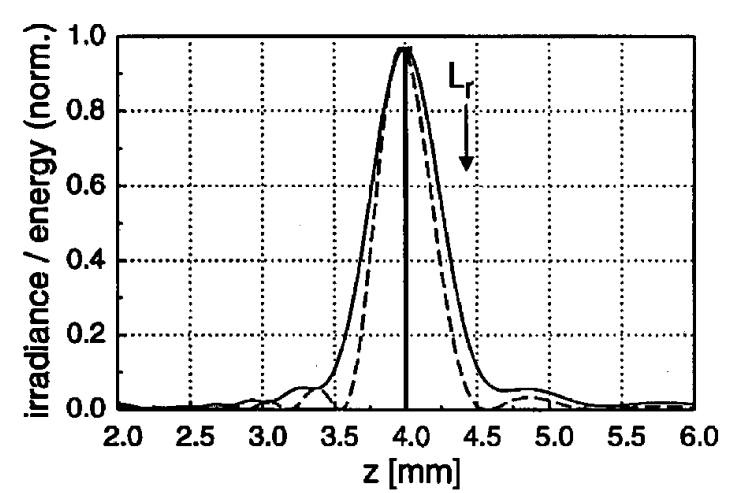

(a) $M=1, Q=8, N A=0.05$

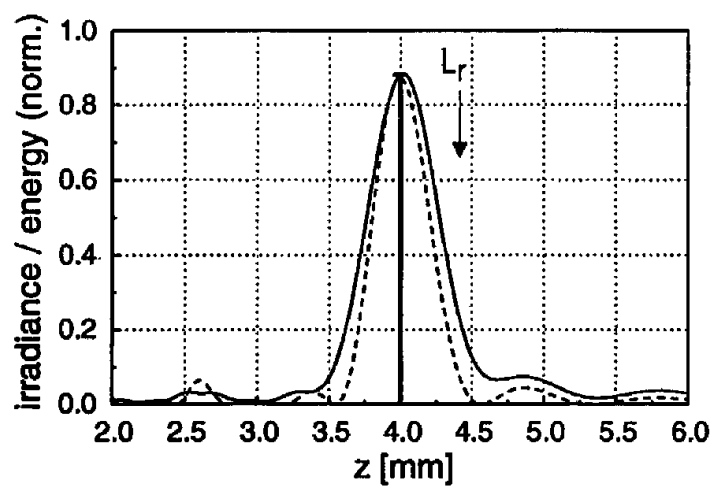

(b) $M=2, Q=4, N A=0.05$

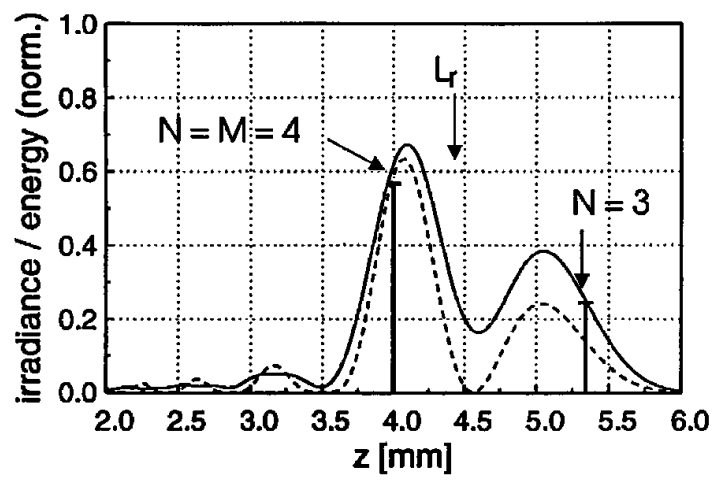

(c) $M=4, Q=2, N A=0.05$

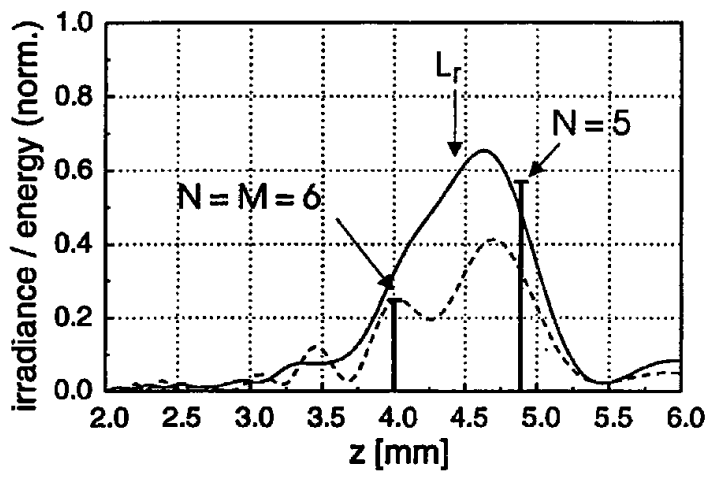

(d) $M=6, Q=2, N A=0.05$

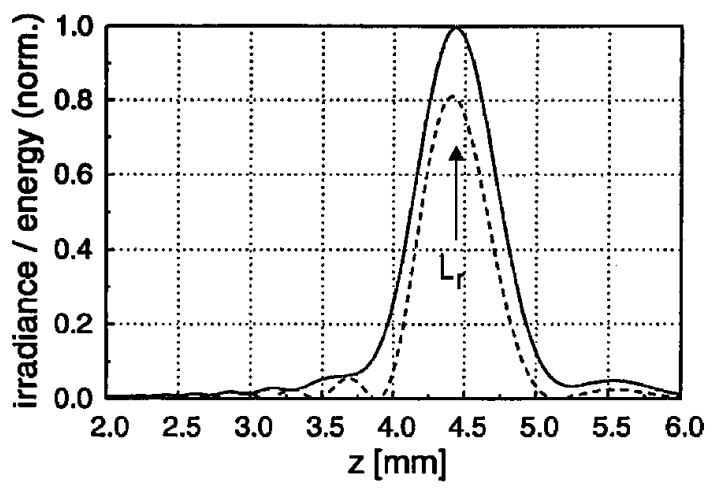

(e) $M=8, Q=1, N A=0.05$

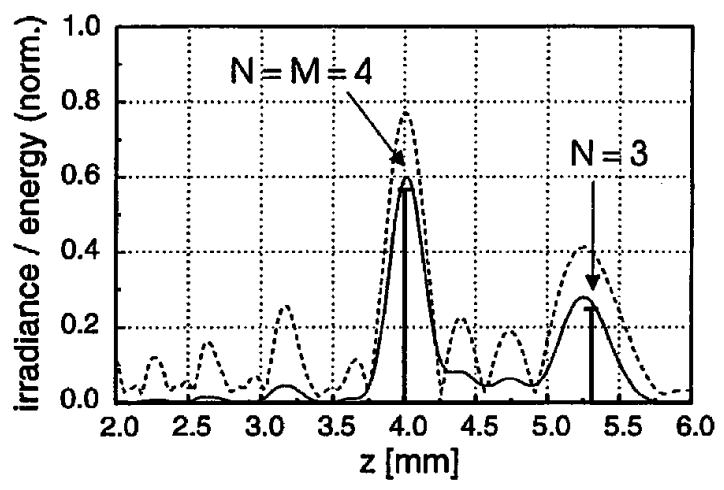

(f) $M=4, Q=4, N A=0.1$

Fig. 5. Calculated distributions of irradiance (dashed curves) and encircled energy (solid curves) along the optical axis $z=z_{12}$ for PMFE's with (a) $M=1$, (b) $M=2$, (c) $M=4,(\mathrm{~d}) M=6$, and (e) $M=8$, all with a depth scaling factor of $\mu=0.9$; (f) the curves for an element with $M=4$ and NA $=0.1$. The vertical lines show the positions and the efficiencies of the focal points calculated with Eqs. (11) and (12).

the efficiency of the discrete focal points, which are calculated under the assumption of a large number of illuminated segments with Eqs. (11) and (12).

The irradiance distribution along the optical axis is governed by two effects: (i) the variations with a sinc ${ }^{2}$ dependence for one single focal point as described in Refs. 18 and 19 and (ii) the diffraction of light in higher-order focal points introduced by the surface-relief distortions.

When the surface-relief profile is distorted by a factor $\mu=0.9$, the focus point calculated by the refraction of rays is at $L_{r} \approx 4440 \mu \mathrm{m}$ for all different design variants. However, wave optics determines where constructive interference between the second- ary wavelets of the different segments occurs. For the purely diffractive lens $(M=1, Q=8)$ there is no lower-order focus point near this refractive focus $L_{r}$ (cf. Fig. 3). Consequently, no additional focus with notable efficiency occurs exactly as predicted by the paraxial equations for diffractive lenses. The same applies for the PMFE with $M=2$, where $Q=4$ segments are illuminated. This proves that a PMFE with only four segments still shows the properties of a diffractive optical element. For higher values of $M$, and consequently lower numbers of $Q$, two types of consequences become relevant. First, the axial density of higher- and lower-order focal points is increasing. In the presence of surface-relief errors this can 
lead to a change in the focal length of the element, i.e., an order other than the $M$ th diffraction order can become the most efficient one $[$ cf. Fig. $5(\mathrm{~d})]$. In the purely refractive case (here for $M=8$ ) there is again only one single focus point observed, with an efficiency that approaches $100 \%$ [cf. Fig. 5(e)]. Its position is shifted by $\Delta z=440 \mu \mathrm{m}$, which coincides exactly with the value obtained by fitting of a spherical lens through the distorted relief and subsequent calculation of the geometrical optical focus.

The second consequence of a high phase-matching number is that, in the transition region between refractive and diffractive elements, the assumption of a high $Q$ for expansion (8) is no longer fulfilled. This leads to deviations of the Rayleigh-Sommerfeld diffraction patterns from the results obtained by Eqs. (11) and (12), which are drawn as vertical lines in Figs. $5(a)-5(d)$. Particularly, a deviation of the axial position of maximum encircled energy from the focal point $L_{\text {par }}$ calculated with the paraxial equations is observed. For the case with $M=1$ and $Q=8$ the position of maximum energy $L_{\mathrm{RS}}$, obtained with the RayleighSommerfeld integrals, exactly coincides with $L_{\mathrm{par}}$. For $M=2(Q=4)$ the position of maximum energy is shifted by $\Delta z_{2}=L_{\mathrm{RS}}-L_{\mathrm{par}}=30 \mu \mathrm{m}$ in the direction toward the refractive focus $L_{r}$. These deviations increase for lower numbers $Q$ of illuminated segments (e.g., $\Delta z_{4}=100 \mu \mathrm{m}$ and $\Delta z_{6}=-180 \mu \mathrm{m}$, both having $Q=2$.

To demonstrate that the transition between refractive and diffractive behavior depends on the number of illuminated segments only, we performed the same simulations for an element with $M=4$ and a doubled aperture size (i.e., $Q=4$ ). The axial diffraction pattern and energy distribution of this PMFE with four illuminated segments are compared with those of the element with $M=4$ and $Q=2$ [cf. Figs. 5(c) and 5(f)], whereas in the case $Q=2$ the behavior differs significantly from the one of a diffractive lens; for $Q=$ 4 a good coincidence with the results of a paraxial diffractive lens is obtained.

In contrast to the energy and the axial position of their maxima, the width of the focal spots showed no change. In Table 1 the FWHM values of the foci for

Table 1. Focus Width (FWHM ${ }^{a}$ values) for Phase-Matched Fresnel Elements with Varying Values for $M$ and $\mu$

\begin{tabular}{cccc}
\hline $\begin{array}{c}\text { Phase- } \\
\text { matching } \\
\text { Number } M\end{array}$ & $\begin{array}{c}\text { Focus } \\
\text { Position } \\
(\mu \mathrm{m})\end{array}$ & $\begin{array}{c}\text { Depth } \\
\text { Scaling } \\
\text { Factor } \mu\end{array}$ & $\begin{array}{c}\text { Focus } \\
\text { Width } \\
(\text { FWHM })\end{array}$ \\
\hline $1^{b}$ & 4000 & 1.00 & 6.7 \\
1 & 4006 & 0.90 & 6.7 \\
2 & 4026 & 0.90 & 6.7 \\
3 & 4038 & 0.90 & 6.7 \\
4 & 4108 & 0.90 & 6.9 \\
8 & 4436 & 0.90 & 7.5 \\
$8^{c}$ & 4440 & 1.00 & 7.5 \\
\hline
\end{tabular}

${ }^{a}$ The theoretical FWHM value for an Airy disk with $q_{a}=7.7 \mu \mathrm{m}$ is $q_{\mathrm{FWHM}} \approx 6.6 \mu \mathrm{m}$.

${ }^{b} \mathrm{PMFE}$ with $\mu=1.0$.

${ }^{c}$ PMFE with $M=8$, designed for $l^{\prime}=4440 \mu \mathrm{m}$. different PMFE's are compared. The widths for $M=$ 1 are equal in the two cases $\mu=1.0$ and $\mu=0.9$. For higher values of $M$ an increasing spot width is observed besides the focal shift. But a comparison with the FWHM of an undistorted element that was designed for the shifted focus position proves that this spot broadening occurs soley because of longer focal length.

\section{Brief Discussion of Polychromatic Applications}

The treatment of polychromatic applications is limited to the case of $\lambda_{1} \neq \lambda_{0}$. A full discussion of the behavior under broad-band illumination would go beyond the scope of this paper. In the paraxial domain, Eqs. (11) and (12) are with a factor $\alpha$ given by Eq. (4). The positions $L_{N}$ of constructive interference and especially the designed diffraction order $M$ are shifted on the $z$ axis owing to the chromatic aberrations of diffraction gratings. The refractive focus, which would be obtained by geometrical optics and which determines the energy distribution among the different diffraction orders, remains at the originally designed position, apart from a small deviation that is due to material dispersion. In analogy to the case of surface distortions, this mismatch results in a reduction of the efficiency in the main focal point and a rise of the energy in the higher or lower diffraction orders. According to previous considerations, the focal point $L_{N}$ situated nearest to the refractive focus will be the most efficient one.

For small values of wavelength deviations $\Delta \lambda=$ $\lambda_{1}-\lambda_{0}$, only a small decrease of efficiency will occur in all various design variants. A value of $\Delta \lambda=70 \mathrm{~nm}$ would be needed for a factor $\alpha=0.9$ in order to change significantly the energy distribution among the different diffraction orders. More important is the axial chromatic aberration, which is linear to $\Delta \lambda$ for small values of $\alpha$. Numerical simulations similar to those presented in Subsection 4.B revealed that this diffractive effect became dominant as soon as more than two secondary wavelets interfered. Figure 6(a) shows that the transition between the purely diffractive and the purely refractive chromatic behavior occurs between the cases of $M=4$ and $M=7$, i.e., when only two segments are illuminated. Furthermore, the encircled energy in a focal point decreases, the higher the absolute value of $\Delta \lambda$ is [cf. Fig. 6(b)]. As in Section 3 , it could be demonstrated that the transition effects shown in Fig. 6 depend only on the number of illuminated segments and not on the absolute value of $M$.

For the case of a high number of illuminated segments (i.e., $Q \geq 4$ ) the efficiency in the higher or lower diffraction orders can be calculated with Eq. (12). For a suitable choice of the values of $\Delta \lambda, M$, and $N$ the diffraction orders can be moved to the originally designed focal point $L^{\prime}$ with $100 \%$ efficiency. This case was described under the name multipleorder diffractive lenses in Ref. 20 and allows the achromatization of planar lenses for a series of discrete wavelengths. The achromatization for a con- 


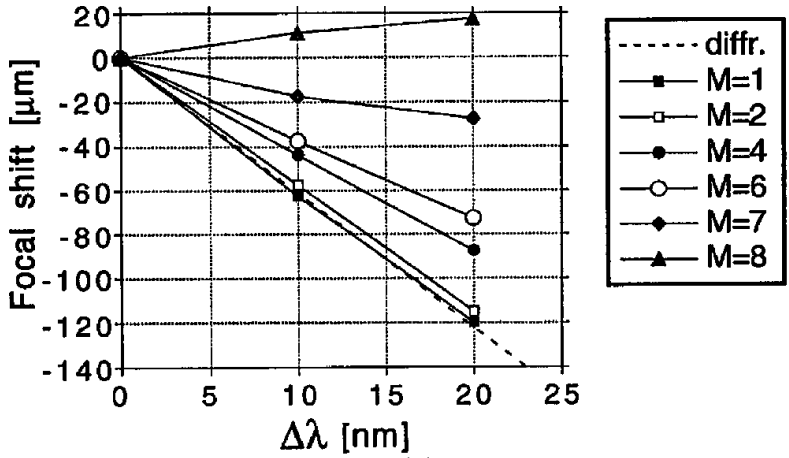

(a)

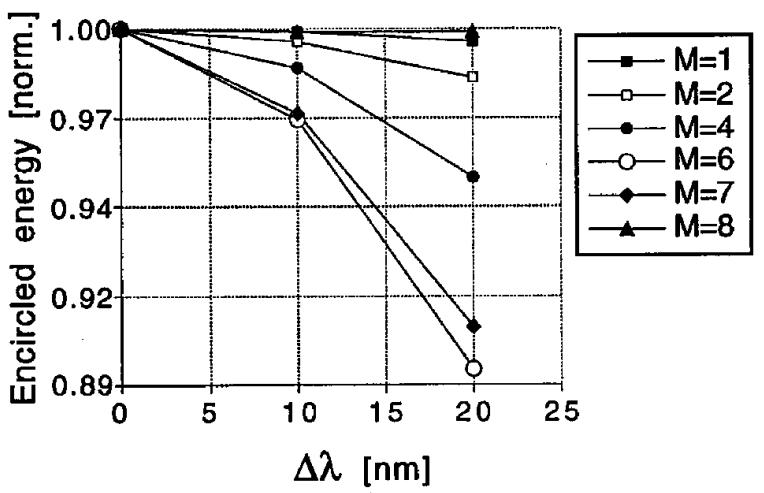

(b)

Fig. 6. Wavelength dependence of (a) focal length $l^{\prime}$ and (b) encircled energy. The dashed line in (a) shows the values for a purely diffractive lens calculated with Eq. (11).

tinuous, broad-band wavelength range with diffractive optics is described in Subsection 4.D.

\section{Achromatization of Classical Lenses}

The previous paragraphs showed that the refractive properties of planar microlenses become important when only a few segments are illuminated and when the incoming light has an extended spectrum. An example in which the exact distinction between refractive and diffractive behavior is of great importance is the hybrid achromat, which combines a refractive lens with a diffractive lens. The two lenses can be combined in one lens, as shown in Fig. 7. The

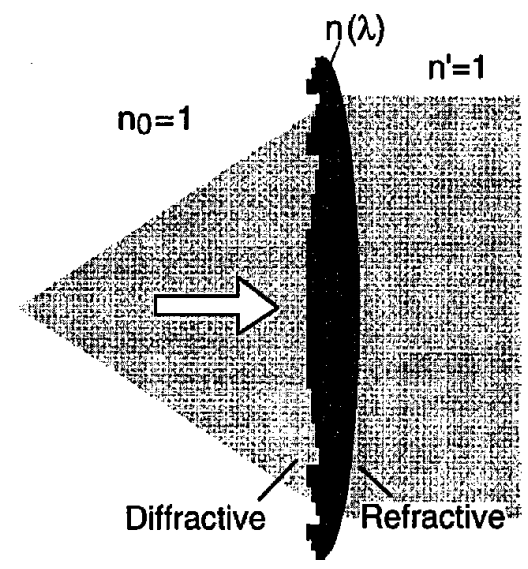

Fig. 7. Hybrid (refractive-diffractive) lens. advantage of such a system is that the axial chromatic aberration of the refractive component can be corrected by a diffractive component with positive focusing power owing to the negative dispersion of the DOE. In a traditional achromatic doublet a refractive element with negative focusing power is needed. Consequently, the required focusing power of the refractive component is greatly reduced in the case of the hybrid lens, thus reducing the magnitude of the higher-order aberrations.

The design of a hybrid achromat is based on geometrical ray tracing. For calculating the influence of the diffractive surface the law of refraction is replaced by the law of grating diffraction. ${ }^{21}$ This approach implies that the diffractive element shows a purely diffractive chromatic behavior, as it corresponds to the curves $M=1$ and $M=2$ in Fig. 6. In the following we use paraxial equations for estimating the critical dimensions of a hybrid achromat.

The focal length $f$ of a thin doublet is given by ${ }^{22}$

$$
\frac{1}{f}=\frac{1}{f_{r}}+\frac{1}{f_{d}},
$$

where in our case $f_{r}$ and $f_{d}$ are the focal lengths of the refractive and the diffractive lenses, respectively.

The condition for an achromat is then ${ }^{23}$

$$
f_{r} v_{r}+f_{d} v_{d}=0
$$

where the dispersions of the refractive lens and the diffractive lens are characterized by the Abbe numbers $v_{r}$ and $v_{d}$, respectively. For a refractive lens the Abbe number is given by

$$
v_{r}=\frac{n_{1}-1}{n_{2}-n_{3}},
$$

where $n_{i}$ are the refractive indices for the wavelengths $\lambda_{i}$. Equation (15) requires that the focal length $f$ of the achromat be the same for the wavelengths $\lambda_{2}$ and $\lambda_{3}$. The central wavelength is $\lambda_{1}$. The definition requires that $\lambda_{2}<\lambda_{1}<\lambda_{3}$. In the case of a diffractive lens the optical power is proportional to the wavelength; therefore the Abbe number is given by

$$
v_{d}=\frac{\lambda_{1}}{\lambda_{2}-\lambda_{3}} .
$$

The Abbe number of a refractive lens is positive, i.e., $v_{r}>0$, whereas for a diffractive lens $v_{d}<0$.

From Eqs. (1) and (2) we can calculate $f_{d}$ as

$$
f_{d}=f\left(1-\frac{v_{r}}{v_{d}}\right) .
$$

The phase function $\Phi(r)$ of a thin diffractive lens with focal length $f_{d}$ is described by

$$
\Phi(r)=\frac{2 \pi}{\lambda_{1}} \frac{r^{2}}{2 f_{d}} .
$$


For a lens of radius $a$ the number $Q$ of illuminated segments is then given by

$$
Q=\frac{\Phi(a)}{2 \pi M}=\frac{1}{\lambda_{1}} \frac{a^{2}}{2 f_{d} M} .
$$

From Eqs. (18) and (20) we obtain an estimation for the number $Q$ of illuminated segments of the diffractive component. $Q$ is a function of the focal length $f$ and the $f$-number $(f / \#=f / 2 a)$ of the hybrid lens, namely,

$$
Q=\frac{f}{8 \lambda M(f / \#)^{2}} \frac{v_{d}}{v_{d}-v_{r}} .
$$

Figure 8 shows a graphical representation of Eq. (21). The example uses an optical glass BK7 and is designed for the wavelength range of $650 \mathrm{~nm} \pm 25 \mathrm{~nm}$, i.e., $\lambda_{1}=650 \mathrm{~nm}, \lambda_{2}=625 \mathrm{~nm}$, and $\lambda_{3}=675 \mathrm{~nm}$. If only a few segments of the achromat are illuminated, then the achromat will not work properly because the achromatization is based on purely diffractive behavior of the diffractive component. As can be seen in Fig. 6(a), the chromatic aberrations of an element start to differ from the purely diffractive case when less than four segments are illuminated. The number of illuminated segments depends on both the phase-matching number $M$ and the aperture of the element. Figure 8 shows that, even for $M=1$, problems occur already for focal lengths shorter than $10-20 \mathrm{~mm}$. The main reason for the low number of segments $Q$ is that the dispersion of a refractive component is much smaller than the dispersion of a diffractive component $\left(v_{r} \approx 10 v_{d}\right)$. Consequently, the diffractive lens needs only a low optical power to compensate the chromatic aberrations of the refractive component [see Eq. (15)]. Another reason for preferring low values of $M$ in a hybrid achromat is given by efficiency considerations. Owing to the broadband illumination, some light will be diffracted in the unwanted diffraction orders of the diffractive lens. Using Eq. (12) with $\alpha_{2}=1.04$ and $\alpha_{3}=0.96$, we see that the efficiency in unwanted diffraction orders will rise for higher values of $M$.

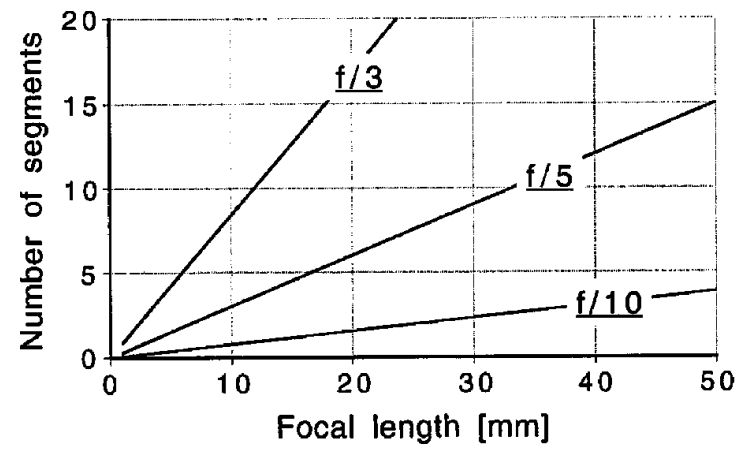

Fig. 8. Hybrid achromat $(M=1)$ : number of diffractive segments versus focal length.

\section{Experimental Results for High-Numerical-Aperture Lenses}

So far, we have treated the case of low-NA lenses, in which the number of illuminated segments can be very low and the purely refractive case is a solution still relevant for planar fabrication techniques. To demonstrate the potential of the PMFE approach, we investigate the effects of small surface distortions for the example of a PMFE with $\mathrm{NA}=0.45(f / \#=1.0)$. The same design wavelength $\lambda_{0}=632.8 \mathrm{~nm}$ as in Subsection 4.4 was chosen; the focal length was set to $l^{\prime}=2.0 \mathrm{~mm}$.

Although the PMFE design procedure is not limited to the paraxial domain, as with many other approaches, and inherently includes the effects of the profile depth, the theoretical maximum efficiency will not reach $100 \%$, as predicted by scalar theory. If we chose $M_{j}=M=1$ for the whole PMFE, a minimum segment width of $w_{\min } \approx 1.3 \mu \mathrm{m}$ would result at the aperture edges. Owing to results based on electromagnetic theory, ${ }^{24}$ structures designed by scalar theory will no longer yield high efficiencies in this regime, and an optimization would need very computing intensive rigorous diffraction calculations. This efficiency reduction also occurs for the larger but deeper segments of an element with $M>1$ and is not yet taken into account in the design procedure.

As in the previous subsections, different design variants were investigated. For the fabrication by laser-beam writing a design strategy was chosen that started with a value of $M_{j}=1$ in the center of the PMFE and increased the phase-matching number $M_{j}$ each time when the minimum segment size for laserbeam writing (here, $w_{\min }=5 \mu \mathrm{m}$ ) was reached. ${ }^{4} \mathrm{~A}$ maximum value of $M_{j}=4$ resulted at a radius of $a=$ $1.0 \mathrm{~mm}$. The diffraction pattern of such a PMFE, fabricated by laser-beam writing in photresist, is shown in Fig. 9. It was measured by scanning of a

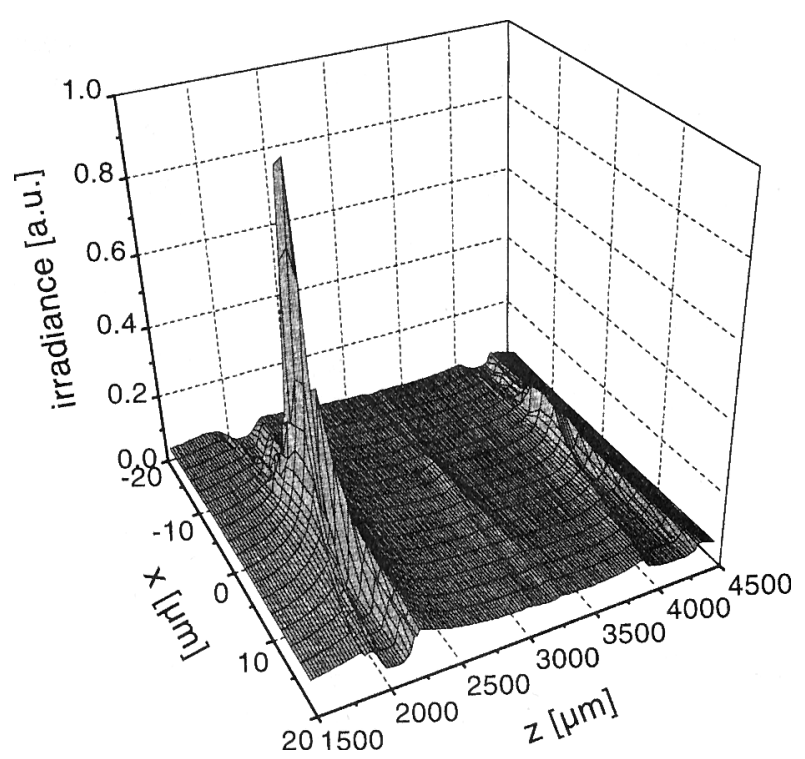

Fig. 9. Measured irradiance distribution in the image space of an $f / 1$ PMFE. 
photodiode masked with a small slit in the $x$ direction at various distances $z$ from the micro-optical element. The FWHM of the spot at a distance of $z_{12}=z=2.0$ $\mathrm{mm}$ was measured to be $\sim 1.5 \mu \mathrm{m}$, compared with the theoretical value of $1.0 \mu \mathrm{m}$ obtained with numerical simulations. We performed the spot-diameter measurements by scanning a pinhole of $2 \mu \mathrm{m} \times 2 \mu \mathrm{m}$ size in the focal plane imaged by a $20 \times$ microscope objective. The deviation of the experimental from the theoretical beam diameter can be explained by inaccuracies in this measurement setup. A maximum efficiency of $\sim 70 \%$ was measured for these PMFE's. The second, small peak at $z \approx 4.0 \mathrm{~mm}$ in Fig. 9 occurs because of deviations in the surface-relief profile and corresponds to a lower diffraction order of the structures with $M_{j}>1$ in the outer regions of the PMFE.

The most important differences in the behavior compared with low-NA lenses can be summarized as follows: First, well-defined higher- and lower-order focal points no longer occur in the irradiance distribution on the optical axis for $\alpha$ and/or $\mu \neq 1.0$. As an illustration, the axial energy distribution of a PMFE with $M=4$ is shown in Fig. 10 . A depth scaling error of $\mu=0.9$ leads to the diffraction of light in the third-order focus, which is spread out on the $z$ axis. The main reason for these large aberrations is that the points $L_{N}$, which fulfill optical phase condition (1) between rays $r_{j}$ and $r_{j+1}$, depend on the radial distance of the rays for lenses with a high NA. An aberrationfree focal point results only for the designed diffraction order $M$ at the design wavelength $\lambda_{0}$ and a perfect surface relief. In addition, steep surface-relief slopes that occur at large radii are much more sensitive to depth errors. With geometrical optical considerations one can see that the focal shift caused by a certain depth error is a monotonically increasing function of the relief slope. A second difference to the behavior of paraxial lenses is that the energy reduction in the main focus owing to $\alpha$ and/or $\mu \neq 1.0$

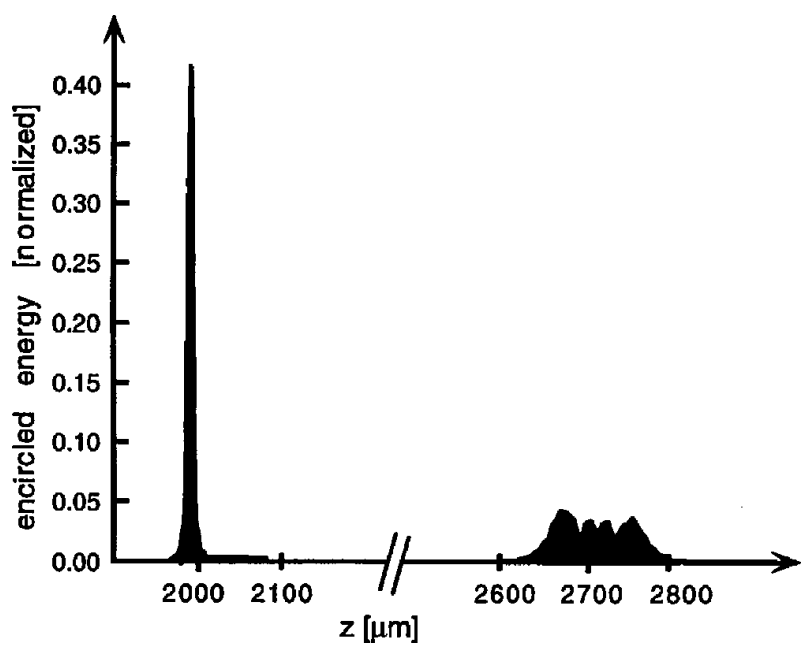

Fig. 10. Calculated axial distribution of the encircled energy of an $f / 1$ lens with a depth scaling factor of $\mu=0.9$. is much higher than predicted by the paraxial equations. In the example of Fig. 10, Eq. (12) would give an efficiency of $\eta_{M}=0.57$ for an error factor $\mu=0.9$, whereas numerical simulations with the RayleighSommerfeld integrals result in a value of $\eta_{M} \approx 0.41$.

\section{E. Discussion}

The results of the above investigations show that diffraction effects are dominant as soon as at least two independent segments of a micro-optical surface relief are illuminated. The laws of wave optics determine the points in the image space where the diffraction at some segment boundary pattern leads to constructive interference. The energy distribution among these diffraction orders is controlled by the phase shift introduced by the relief structure between the segment boundaries and can be obtained by solving the wave equations, e.g., in the RayleighSommerfeld approximation. Nevertheless, the laws of geometrical optics can be used to obtain a rough estimation on the effect of typical fabrication inaccuracies.

In the PMFE design the refractive and the diffractive behavior of planar microlenses can be adjusted by means of the phase-matching number $M_{j}$, mainly in the sense that the size of contiguous surface relief elements can be varied over the elements' area. Elements with different $M_{j}$, whose theoretical optical functions are identical, behave differently when small fabrication errors occur or polychromatic applications are considered. Therefore the design of a specific element has to take into account fabrication constraints, as well as the most important requirements of an application. Some design rules can be given for different ranges of numerical apertures, as follows.

For low numerical apertures, elements with a constant $M_{j}$ and $M$ can be designed that have either purely refractive or purely diffractive properties. In the paraxial approximation the number $Q$ of illuminated segments is given by

$$
Q \approx \frac{l^{\prime}(\mathrm{NA})^{2}}{2 M \lambda_{0}}
$$

Therefore with a suitable choice of $M$ both types of behavior are accessible: either a refractive element with low chromatic aberrations, but with a focus position that is strongly dependent on the exact surface-relief profile, or a diffractive planar optical element showing large chromatic aberrations, but with a focus position that is not very sensitive on the surface-relief profile. For any practical realization the upper limit for $M$ is given by the maximum available profile depth, whereas the lateral resolution imposed by the fabrication technology determines the minimum value of $M$. The different design examples of a NA $=0.05$ lens with $l^{\prime}=4 \mathrm{~mm}$ that are presented in this section represent the actual limits for the fabrication by direct laser-beam writing. 
As soon as more than a few segments of planar microlenses are illuminated (a typical number is $Q \geq 4$ ), their behavior under typical fabrication and wavelength inaccuracies corresponds to a diffractive optical element. Nevertheless, the variation of the parameter $M_{j}$ offers some important perspectives for the fabrication and also for the design of new types of elements. As an advantage of the diffractive behavior, the focal position of such microlenses is not affected by small surface-relief errors, whereas chromatic aberrations are very strong. However, elements with a high $M$ have an increased density of possible focal points along the $z$ axis and therefore can react with an axial shift of the most efficient focal point in the presence of fabrication errors concerning the relief depth. Nevertheless, these designs are not necessarily worse than the case of $M=1$. The most important consequences of a design with a high value of $M$ are the larger lateral feature sizes and consequently the lower lateral resolution required for their realization. For many technologies, e.g., laser-beam writing, the fabrication of high-NA elements is made possible only with the PMFE concept. In addition, a reduction of optical losses may be achieved by the lower number of segment boundaries, which are the main sources for stray light. A potential drawback of elements that use the full depth available by a high $M$ may be the need for a higher relative fabrication accuracy in the vertical dimension. Because for all interference effects (be it in the first or the $M$ th order) the relative optical path differences in fractions of the wavelength $\lambda_{0}$ are relevant, each profile step at a segment boundary requires the same absolute depth accuracy.

\section{Conclusions}

The refractive and the diffractive properties of planar micro-optical elements were investigated for the example of phase-matched Fresnel elements. Their design optimizes locally the surface-relief profile, takes into account the finite profile depth, and is limited only by the validity of the scalar theory. By the variation of the phase-matching number the width and the depth of the surface-relief segments can be adjusted locally. The optical properties of a series of design variants were compared. By variation of the number of illuminated segments at a constant numerical aperture the transition between purely refractive and diffractive lenses was numerically simulated. While all these designs perform exactly the same optical function in theory, their refractive or diffractive characteristic becomes obvious when fabrication inaccuracies or wavelength deviations occur. The main result of the numerical simulations in the Rayleigh-Sommerfeld approximation is that the diffractive behavior of a planar microlens is dominant when more than approximately four segments are illuminated. In the paraxial region their behavior under typical fabrication and application conditions can then be described by the use of a Fourier series, which is identical to a sum over refractive lenses with different focal points and efficiencies. For a number of different applications, including low- and high-NA lenses and hybrid achromats, fabrication-oriented design rules have been given.

The authors gratefully acknowledge helpful discussions with R. Dändliker and M. T. Gale. This work was supported in part by the Swiss Priority Program OPTIQUE of the Board of the Swiss Federal Institute of Technology.

\section{References}

1. M. Born and E. Wolf, Principles of Optics (Pergamon, London, 1989), Chap. 3.1.

2. R. E. Kunz and M. Rossi, "Phase-matched Fresnel elements," Opt. Commun. 97, 6-10 (1993).

3. W. T. Welford, Aberrations of Optical Systems (Hilger, London, 1986), Chap. 2.1

4. M. Rossi, R. E. Kunz, and G. L. Bona, "Phase-matched Fresnel elements for optical interconnects," in Diffractive Optics, Vol. 11 of 1994 OSA Technical Digest Series (Optical Society of America, Washington, D.C., 1994), pp 321-324.

5. J.A. Futhey and M. Fleming, "Superzone diffractive lenses," in Diffractive Optics: Design, Fabrication and Applications, Vol. 9 of 1992 OSA Technical Digest Series (Optical Society of America, Washington, D.C., 1992), pp 9-6.

6. J. C. Marron, D. K. Angell, and A. M. Tai, "Higher-order kinoforms," in Computer and Optically Formed Holographic Optics, I. Cindrich and S. H. Lee, eds., Proc. Soc. Photo-Opt. Instrum. Eng. 1211, 62-66 (1990).

7. D. A. Buralli, G. M.Morris, and J. R. Rogers, "Optical performance of holographic kinoforms," Appl. Opt. 28, 976-983 (1989).

8. L. N. Hazra, Y. Han, and C. Delisle, "Plane kinoform lenses for axial stigmatism in finite conjugate imaging," Opt. Commun. 91, 1-4 (1992).

9. J. S. Fender and R. A. Carreras, "Demonstration of an optical phased telescope array," Opt. Eng. 27, 706-711 (1988).

10. M. T. Gale, M. Rossi, J. Pedersen, and H. Schütz, "Fabrication of continuous-relief micro-optical elements by direct laser writing in photoresist," Opt. Eng. 33, 3556-3566 (1994).

11. D. Zaleta, W. Daschner, M. Larsson, B. C. Cress, J. Fan, K. S. Urquhart, and S. H. Lee, "Diffractive optics fabricated by electron-beam direct write methods," in Diffractive and Miniaturized Optics, S. H. Lee, ed., Proc. Soc. Photo-Opt. Instrum. Eng. CR49, 117-137 (1993).

12. G. J. Swanson and W. B. Veldkamp, "Diffractive optical elements for use in infrared systems," Opt. Eng. 28, 605-608 (1989).

13. H. Dammann, "Blazed synthetic phase-only holograms," Optik 31, 95-104 (1970).

14. J. W. Goodman, Introduction to Fourier Optics (McGraw-Hill, New York, 1968), Chap. 5.1.

15. S. Sinzinger, M. Testorf, and W. Singer, "The transition between diffractive and refractive micro-optical components," in Diffractive Optics, Vol. 11 of 1994 OSA Technical Digest Series (Optical Society of America, Washington, D.C., 1994), pp. 143-146.

16. J. D. Gaskill, Linear Systems, Fourier Transforms, and Optics (Wiley, New York, 1978), Chap. 10.2.

17. K. Miyamoto, "The phase Fresnel lens," J. Opt. Soc. Am. 51, 17-20 (1961).

18. J. J. Stamnes, Waves in Focal Regions (Hilger, London, 1986), Chap. 12.1 
19. Ref. 1, Chap. 8.8.

20. G. M. Morris and D. Faklis, "Achromatic and apochromatic diffractive singlets," in Diffractive Optics, Vol. 11 of 1994 OSA Technical Series (Optical Society of America, Washington, D.C., 1994), pp. 53-56.

21. Ref. 3, Chap. 11.3.
22. Ref. 3, Chap. 10.2

23. E. Hecht and A. Zajac, Optics (Addison-Wesley, Reading, Mass., 1987), Chap. 6.3.

24. E. Noponen, J. Turunen, and A. Vasara, "Electromagnetic theory and design of diffractive-lens arrays," J. Opt. Soc. Am. A 10, 434-443 (1993). 